\title{
Magnetic Digital Microfluidics - A Review
}

Yi Zhang $^{1 *}$ and Nam-Trung Nguyen ${ }^{2}$

${ }^{1}$ School of Mechanical and Aerospace Engineering, Nanyang Technological University, Singapore

${ }^{2}$ Queensland Micro- and Nanotechnology Centre, Griffith University, Brisbane, QLD 4111, Australia

*Correspondence should be addressed to Y. Zhang: yi_zhang@ntu.edu.sg. 


\begin{abstract}
A digital microfluidic platform manipulates droplets on an open surface. Magnetic digital microfluidics utilizes magnetic forces for actuation and offers unique advantages compared to other digital microfluidic platforms. First, the magnetic particles used in magnetic digital microfluidics have multiple functions. In addition to serving as actuators, they also provide a functional solid substrate for molecule binding, which enables a wide range of applications in molecular diagnostics and immunodiagnostics. Second, magnetic digital microfluidics can be manually operated in a "powerfree" manner, which allows for operation in low-resource environments for point-of-care diagnostics where even batteries are considered a luxury item. This review covers research areas related to magnetic digital microfluidics. This paper first summarizes the current development of magnetic digital microfluidics. Various methods of droplet manipulation using magnetic forces are discussed, ranging from conventional magnetic particle-based actuation to the recent development of ferrofluids and magnetic liquid marbles. This paper also discusses several new approaches that use magnetically controlled flexible substrates for droplet manipulation. In addition, we emphasize applications of magnetic digital microfluidics in biosensing and medical diagnostics, and the current limitations of magnetic digital microfluidics are identified. We provide a perspective on possible solutions to close these gaps. Finally, the paper discusses the future improvement of magnetic digital microfluidics to explore potential new research directions.
\end{abstract}




\section{Introduction}

Digital microfluidics has been established as a research field of its own for a decade. Together with continuous-flow droplet microfluidics, digital microfluidics lays the foundation for droplet-based microfluidics. Continuous-flow droplet-based microfluidics, also known as emulsion microfluidics, deals with pico- to nanolitre droplets that are continuously generated in a closed microfluidic network. These droplets are primarily manipulated in closed microchannels. Usually, many droplets are generated that are not manipulated individually. ${ }^{1-4}$ Continuous-flow droplet-based microfluidics has been adopted for high-throughput parallel reactions such as digital polymerase chain reaction (PCR) and sequencing library preparation. In contrast, digital microfluidics deals with discrete nanoto microlitre droplets. ${ }^{5-8}$ The droplets are manipulated on a plain surface with no confinement or in an open channel or well with partial confinement. These discrete droplets on an open-surface platform are usually individually controlled and act as virtual reaction chambers. This approach is often used in point-of-care diagnostics that require complex sample preparation ${ }^{9-17}$ or in on-demand synthesis of hazardous materials. ${ }^{18-20}$ In this review, we refer to the open-surface droplet platform as digital microfluidics.

Based on the actuation mechanism, digital microfluidics can be further categorized into electrowetting on dielectric (EWOD), ${ }^{5,721-23}$ magnetic, $^{9,12,13,24-26}$ surface acoustic wave (SAW) $)^{27-30}$ and other types ${ }^{31-34}$. Currently, EWOD is the most popular actuation concept, followed by magnetic and SAW. EWOD is very beneficial for droplet manipulation and offers automated and precise droplet control. Most importantly, EWOD is able to split and dispense droplets with great ease. Many recent advances have overcome earlier limitations of the EWOD platform. EWOD-based digital microfluidic platforms can now be applied for more complex operations and more intricate bioassays. To date, several reviews have covered various aspects of EWOD-based digital microfluidics. $5,22,23$

Magnetic actuation is less popular than EWOD but its unique advantages should not be overlooked. Tab. 1 summarizes and compares the features of magnetic digital microfluidics and EWOD-based digital microfluidics. Conventionally, magnetic digital microfluidics manipulates droplets by controlling magnetic particles in the droplet using permanent magnets or electromagnets. The magnetic particles in turn drag the droplet along. The most unique feature of magnetic digital microfluidics is the dual functionality of magnetic particles. In addition to their role as the droplet actuator, the magnetic particles also provide a functional solid substrate for molecule adsorption. Several papers have demonstrated that silica-functionalized magnetic particles can control droplet motion and bind DNA molecules for solid phase DNA extraction. ${ }^{10-13}$ The chemical function of magnetic particles makes magnetic digital microfluidics very attractive for droplet-based 
bioassays. EWOD-based digital microfluidic platforms often employ functional magnetic particles for complex bioassays, ${ }^{35-37}$ thus requiring the introduction of magnetic control or other separation mechanisms $^{38,39}$ in addition to the primary droplet actuation mechanism. In contrast, magnetic digital microfluidics uses particles for both actuation and biochemical assays, which greatly reduces the complexity of the platform.

In a two-plate EWOD system, the droplets are sandwiched between two flat surfaces separated by a small distance. Therefore, the droplet size is limited to several picolitres to hundreds of nanolitres. A magnetic digital microfluidic platform handles droplets of submicrolitres to tens of microlitres, which is larger than those employed in the two-plate EWOD. When testing clinical samples, targets of interest are usually present at low concentrations, and there is a mismatch between the required sample volume and the volume that a microfluidic system can handle. ${ }^{40-42}$ The magnetic digital microfluidic platform allows for a larger sample volume and can potentially achieve a higher detection sensitivity. Single-plate EWOD system ${ }^{43}, 44$ is capable of handling droplets of a relatively large volume comparable to that of magnetic digital microfluidics. Nonetheless, singleplate EWOD cannot perform liquid dispensing and droplet splitting, ${ }^{5}$ thereby losing tremendous advantages of the EWOD-based system. The liquid volume handled by a magnetic digital microfluidic platform is highly scalable. The same platform could operate with droplet from submicrolitre to tens of microlitres. Even a large volume would be possible except the fact that the liquid forms a puddle at such large volumes because the gravity becomes dominant over the capillary force. Nevertheless, one may still use magnetic particles to manipulate this large liquid puddle.

Both magnetic digital microfluidics and EWOD-based digital microfluidics use a hydrophobic lowfriction substrate for droplet manipulation, but the substrate used by magnetic digital microfluidics has a much simpler structure. Conventional magnetic digital microfluidics only needs a plain surface coated with a hydrophobic material such as Teflon AF. More advanced magnetic digital microfluidic platform with assistive features may have additional physical structures or chemical modifications. However, these features only add one additional layer to the substrate. The basic EWOD substrate is more complex and requires multiple layers of structures. Let's take a two-plate EWOD system for example, the bottom plate has a dielectric layer to cover the electrodes, and the hydrophobic layer is coated on top of the dielectric layer. On the top plate, a transparent indium tin oxide (ITO) layer is coated on the substrate followed by another hydrophobic layer on top. Despite the fact that the use of printed circuit board (PCB) as the EWOD substrate greatly simplifies the fabrication process, the EWOD substrate is still considerably costlier than a plain substrate used in magnetic digital microfluidics. 
Magnetic digital microfluidics provides a much more flexible fluidic control. Compared to conventional channel-based microfluidics where fluids flow in pre-defined path, the fluidic path in digital microfluidics is virtual and reprogrammable. Therefore, a single digital microfluidic design can be used for multiple purposes. Magnetic digital microfluidics is more flexible than EWOD-based microfluidics. Although the droplet path can be reprogrammed on a EWOD platform, the path must follow the pre-fabricated electrode pattern. In contrast, magnetic digital microfluidics does not need to follow any pre-defined surface pattern, except in some cases of assisted magnetic digital microfluidics where droplets need to pass through pre-defined assistive features on the substrate. In addition, Magnetic digital microfluidic platforms are compatible with automated control systems and can be operated manually or by other means that do not require an external power source, which is an important consideration for point-of-care applications in resource-limited environments where electricity is a luxury item.

Another major advantage of magnetic digital microfluidics compared to EWOD is its relatively higher tolerance to liquid properties. The driving force by EWOD is highly dependent on the surface tension, the conductivity and permittivity of the liquid that forms the droplet. Manipulating droplets with low conductivity and permittivity using EWOD requires excessively high driving voltage. ${ }^{5}$ Although magnetic droplet manipulation is also influenced by the properties of the liquid, the conditions can be easily adjusted to ensure a smooth droplet operation. For example, for liquid with low surface tension or high viscosity, more magnetic particles and a slower moving speed may be used to ensure that droplets move with magnetic particles rather than split with the magnetic particles or disengage with the magnet.

The dual functionality of magnetic particles that simplifies droplet manipulation, the flexible fluidic control and the electrical "power-free" operation give magnetic digital microfluidics unique advantages over other systems. Because of the unique advantages of magnetic digital microfluidics, we believe its significance should not be overlooked (Fig. 1). The simple operation has made magnetic digital microfluidic platforms well-suited for many sample-to-answer applications. Nonetheless, compared to EWOD, magnetic digital microfluidics has several limitations that must be addressed to further extend its applicability. This review first summarizes the current development of magnetic digital microfluidics with a focus on recent advances in new magnetic actuation concepts. We also emphasize applications of magnetic digital microfluidics in biosensing and biomedical diagnostics. We then identify the current limitations of magnetic digital microfluidics and suggest possible solutions for better droplet control and broader applications. Finally, we discuss methods for improving the functionality of magnetic digital microfluidics to explore new research directions. 
Page 5 


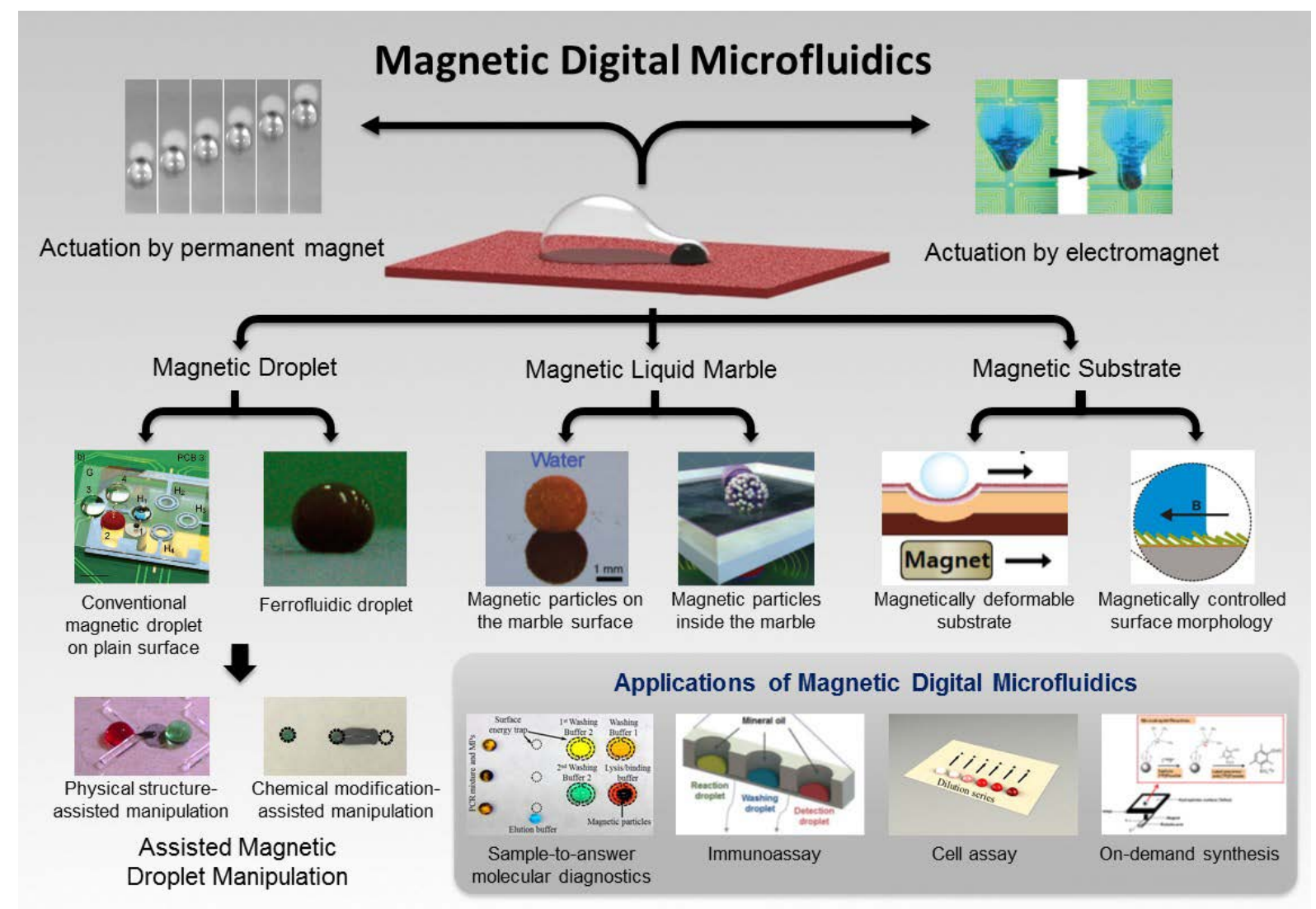

Fig. 1 An overview of magnetic digital microfluidics. Inset images reproduced from Ref. 12, 25, 45-48 with permission from the Royal Society of Chemistry, from Ref. 13, 49, 50 with permission from Wiley, and from Ref. 18, 50, 51 with permission from the American Chemical Society.

Tab. 1 Comparison of magnetic digital microfluidics and EWOD-based digital microfluidics. 


\begin{tabular}{|c|c|c|}
\hline & Magnetic Digital Microfluidics & EWOD-based Digital Microfluidics \\
\hline \multicolumn{3}{|l|}{ Actuation } \\
\hline $\begin{array}{l}\text { Actuation } \\
\text { mechanism }\end{array}$ & $\begin{array}{l}\text { Magnetic force controlled by } \\
\text { magnetic particles or magnetic } \\
\text { substrate as well as applied } \\
\text { magnetic field }\end{array}$ & $\begin{array}{l}\text { Pressure gradient as a result of } \\
\text { change in contact angle controlled } \\
\text { by applied electrical potential }\end{array}$ \\
\hline \multirow{2}{*}{ System control } & $\begin{array}{c}\text { Automation by translation stage or } \\
\text { patterned electromagnets }\end{array}$ & $\begin{array}{c}\text { Efficient automation with patterned } \\
\text { electrode }\end{array}$ \\
\hline & $\begin{array}{l}\text { Manual operation possible in an } \\
\text { electrical "power-free" manner }\end{array}$ & Manual operation is not feasible \\
\hline Substrate & $\begin{array}{c}\text { Simple substrate with a } \\
\text { hydrophobic coating or one } \\
\text { additional layer for assitive features }\end{array}$ & $\begin{array}{c}\text { Mulitple layers of structures } \\
\text { including electrode layer, dielectric } \\
\text { layer, transparent conductive layer } \\
\text { and hydrophobic coating }\end{array}$ \\
\hline \multicolumn{3}{|l|}{ Droplet } \\
\hline \multirow[b]{2}{*}{ Size } & \multirow[b]{2}{*}{ Submicrolitre to tens of microlitres } & Two-plate: picolitres to microlitres \\
\hline & & $\begin{array}{l}\text { One-plate: submicrolitres to tenso } \\
\text { fo microlitres }\end{array}$ \\
\hline Liquid properties & $\begin{array}{l}\text { Influenced by the surface tension } \\
\text { of the fluid, but relatively more } \\
\text { tolerable to liquid properties }\end{array}$ & $\begin{array}{l}\text { Relatively more sensitive to the } \\
\text { surface tension, conductivity and } \\
\text { permittivity of the fluid }\end{array}$ \\
\hline \multicolumn{3}{|l|}{ Fluidic Operation } \\
\hline Moving and Merging & Efficient & Efficient \\
\hline Particle extraction & $\begin{array}{l}\text { No additional actuation mechanism } \\
\text { is required, magnetic force alone is } \\
\text { sufficient for particle extraction }\end{array}$ & $\begin{array}{l}\text { Additional mechanism is required } \\
\text { to extract particles from the droplet }\end{array}$ \\
\hline Mixing & $\begin{array}{l}\text { Passive mixing, or active mixing } \\
\text { with additional components }\end{array}$ & Efficient passive mixing \\
\hline \multirow{2}{*}{$\begin{array}{l}\text { Liquid dispensing } \\
\text { and droplet splitting }\end{array}$} & $\begin{array}{l}\text { Impossible on conventional } \\
\text { magnetic digital microfluidic } \\
\text { platform }\end{array}$ & \multirow{2}{*}{$\begin{array}{l}\text { Efficient liquid dispensing and } \\
\text { droplet splitting on two-plate } \\
\text { EWOD-based digital microfluidic } \\
\text { platform, and the dispensed } \\
\text { droplets move freely }\end{array}$} \\
\hline & $\begin{array}{c}\text { Possible with assisted-magnetic } \\
\text { digital microfluidics, but dispensed } \\
\text { droplet is immobilized }\end{array}$ & \\
\hline Assays & $\begin{array}{l}\text { Magnetic particles function both as } \\
\text { the droplet actuator and the solid } \\
\text { substrate for biochemical reaction }\end{array}$ & $\begin{array}{c}\text { Requires the addtion of particles for } \\
\text { solid phase reactions }\end{array}$ \\
\hline
\end{tabular}




\section{Actuation Mechanisms of Magnetic Digital Microfluidics}

\section{Magnetic Particle-Based Manipulation}

On a magnetic digital microfluidic platform, liquid droplets are manipulated on a natural ${ }^{52}$ or synthetic ${ }^{13}$ hydrophobic surface. In the most conventional magnetic manipulation method, magnetic particles are added to the droplet to serve as the actuator (Fig. $\mathbf{2 a}$ and $\mathbf{2 b}$ ). We collectively refer to ferromagnetic, paramagnetic and superparamagnetic particles as magnetic particles. A permanent magnet or an electromagnet is placed beneath the hydrophobic surface. By moving a permanent magnet or inducing an alternating magnetic field through an electromagnet, one can control the movement of the droplets containing the magnetic particles. One important aspect of magnetic digital microfluidics is the substrate with low surface tension and adhesion, allowing droplets to move with minimum friction. To fabricate a substrate with these properties, the surface of the substrate is coated with materials with low surface energy. The most commonly material is Teflon AF, sometimes with a fluorosaline as a primer layer for improved Teflon AF adhesion. The resulting surface has a low surface energy and is hydrophobic and oleophobic, providing a "slippery" substrate for droplet movement. The quality of the low-surface-energy substrate is not only critical to magnetic digital microfluidics but also to digital microfluidics in general.

Detailed studies have been conducted on the physics governing the motion, coalescence of droplets and particle extraction from droplets. ${ }^{13,45}$ These manipulation tasks are essential for bioanalytical assays, as they are equivalent to performing liquid transfer, and addition and discarding waste liquid in a typical test tube. The movement speed of the permanent magnet and the mass of the magnetic particles play determining roles in the manipulation of magnetic droplets (Fig. 2c) as pointed out by Long et al. ${ }^{45}$ This important study ${ }^{45}$ discussed the fundamentals of conventional magnetic droplet operation and provided design guidelines for magnetic digital microfluidic platforms. This study also points out another important aspect of magnetic digital microfluidics, which is the magnetic particles (or ions in some cases). The droplet manipulation on the magnetic digital microfluidic platform is a combined effect of surface tension, surface friction and magnetic force. The magnetic force is determined by the mass of magnetic particles, magnetic susceptibility of magnetic particles and the strength of the magnetic field. Due to a limited selection of magnetic particles, the magnetic force is often adjusted by using different amounts of magnetic particles or applying magnetic fields of various strengths. No detailed study has been conducted to examine the effect of magnetic susceptibility of the particles on droplet manipulation. An interesting study by Mats et al. investigated the effect of magnetic susceptibility of ionic droplets, i.e. droplets with dissolved paramagnetic salts. In this scenario, it is easier to control the magnetic susceptibility by varying the salt concentration. 

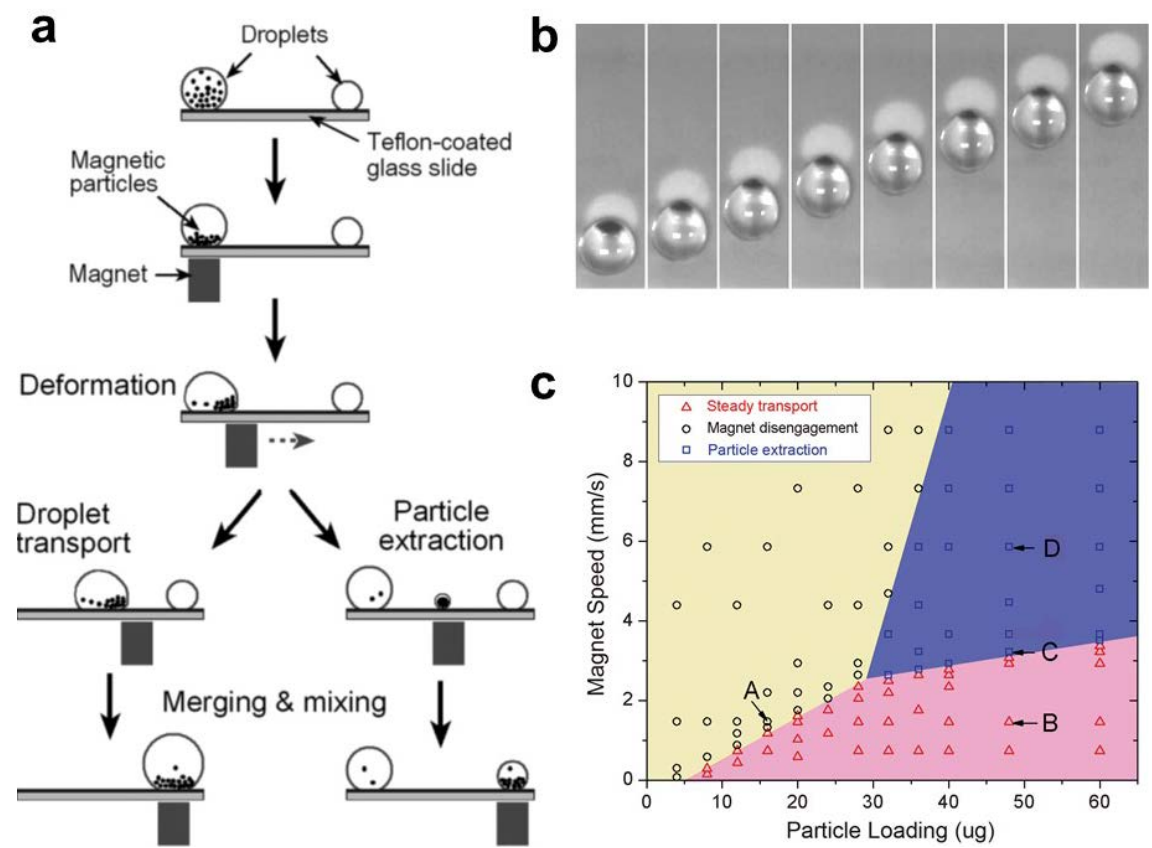

Fig. 2 Conventional magnetic digital microfluidics. a) Principle of conventional magnetic digital microfluidic actuation. b) Serial images of droplets actuated by magnetic particles and a permanent magnet. c) Phase diagram of magnetic digital microfluidic actuation. Reproduced from Ref. 45 with permission from the Royal Society of Chemistry.

Several groups have demonstrated the use of magnetic droplets as virtual reaction chambers for complex bioanalytical assays on magnetic digital microfluidic platforms. Pipper et al. performed sample-to-answer molecular diagnostics of H5N1 avian flu on a magnetic digital microfluidic platform integrated with a miniaturized thermal cycler and a fluorescence-based detection system. ${ }^{10}$ The authors used silica-functionalized magnetic particles to control the droplet motion and to purify RNA from the sample matrix for the detection of target RNA using PCR. The entire sample preparation and PCR-based detection process was implemented using a small permanent magnet. The same group demonstrated a sample-to-answer detection process that combined cell isolation and PCR-based sensing on an automated magnetic digital microfluidic platform. The magnetic particles were controlled by a permanent magnet fixed to a step motor, ${ }^{11}$ and all droplets moved along a circular path (Fig. 3). Interestingly, the PCR was performed in a space domain by moving the droplet between 3 temperature zones using magnetic actuation. Magnetic digital microfluidics is also suitable for other types of heterogeneous assays, i.e., assays that require washing such as ELISA. Shikida et al. and a few other groups presented particle-based ELISA on a magnetic digital microfluidic platform. ${ }^{47,53-55}$ The magnetic particles used for ELISA were functionalized with antibodies to capture the target molecules. Target recognition and particle washing were realized by moving the droplet, extraction the magnetic particles and merging the particles with another droplet. 
Several studies have demonstrated the manipulation of multiple droplets in parallel using an array of magnets for potential high-throughput applications. Ohashi et al. used a linear array of magnets to cycle droplets between three temperature zones for parallel PCR. ${ }^{56}$ Okochi and Shi both demonstrated parallel RNA extraction from multiple samples in a droplet array manipulated by magnetic particles. ${ }^{57,58}$ Park et al. demonstrated particle-based ELISA in parallel using a magnetic droplet array. ${ }^{47}$ In most cases, the permanent magnet was positioned beneath the open surface. In some cases, the permanent magnet was placed next to the droplet on the same plane. For example, Tsuchiya et al. manipulated a droplet in an open channel using a permanent magnet placed in another parallel channel near the droplet channel. ${ }^{59}$
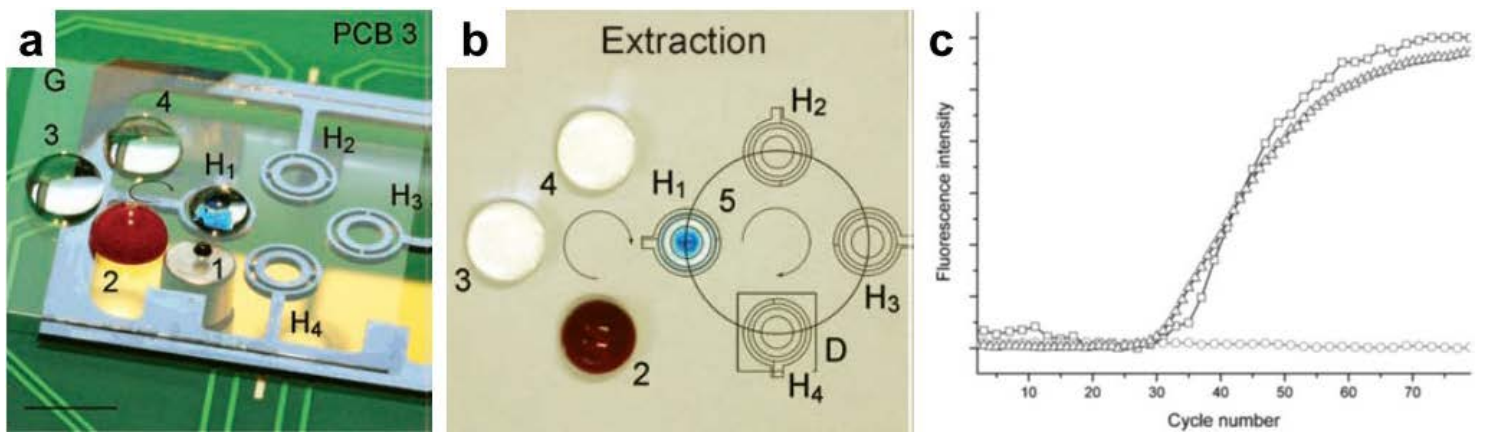

Fig. 3 A magnetic digital microfluidic platform integrated with automated magnetic control, a miniaturized thermal cycler and a fluorescence-based real-time detection system. a) A permanent magnet fixed on a rotational motor controls the droplet motion in a circular path. Droplet 1 functional magnetic particles, Droplet 2 - sample in binding buffer, Droplets 3 and 4 - washing buffer, Droplet 5 - PCR buffer. b) H1, H2, H3 and H4 are four temperature zones. The PCR buffer droplet is magnetically driven between the four temperature zones for thermal cycling. c) Real-time PCR results. Reproduced from Ref. 13 with permission from Wiley. 
In the examples above, the magnetic particles were controlled using a permanent magnet. Another method for controlling the magnetic particles is based on an array of electromagnets consisting of micro coils (Fig. 4). To use electromagnets, a droplet containing magnetic particles is suspended in silicone oil or mineral oil. The micro coils function as electromagnets with controllable strength and polarity and create a small magnetic field gradient to displace the magnetic particles and consequently the droplet. Earlier studies demonstrated micro coil-enabled magnetic droplet manipulation and analytically and numerically investigated the underlying principles. ${ }^{60-62}$ The pattern of the micro coils was optimized to provide efficient 2D droplet operation. ${ }^{63,64}$ Using electromagnets to actuate the droplet, Lehmann et al. ${ }^{26,49}$ demonstrated DNA purification following a process similar to that of Pipper et al. ${ }^{26,49}$ Chiou et al. demonstrated both DNA purification and PCR-based detection. ${ }^{9}$

One major issue associated with electromagnet-based system is Joule heating. On a magnetic digital microfluidic platform, this problem is partly mitigated by the use of oil. The oil required to suspend the droplet also prevents its evaporation. The large thermal mass of the oil prevents the temperature from increasing too rapidly. Chiou et al. added an active cooling device below the electromagnet and managed to keep the temperature below $30^{\circ} \mathrm{C}$ during the entire operation. ${ }^{9}$

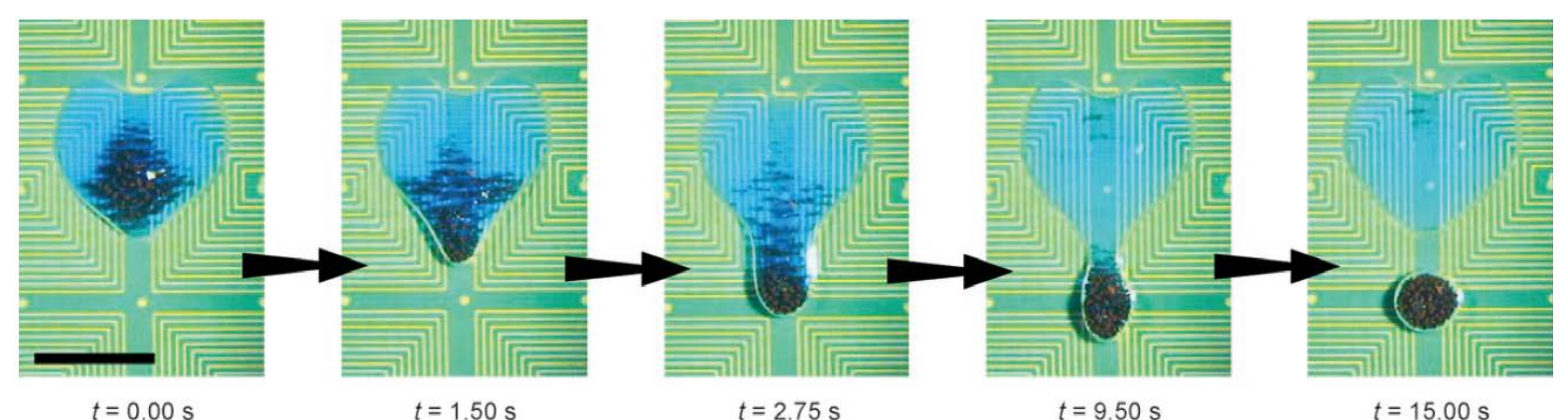

Fig. 4 Magnetic digital microfluidic platform using an electromagnet for droplet manipulation. Reproduced from Ref. 49 with permission from Wiley. 


\section{Ferrofluid-Based Magnetic Droplet Manipulation}

Ferrofluids contain a colloidal suspension of nanoscale magnetic particles. ${ }^{65}$ The particles are coated with surfactant and remain colloidally stable in the liquid. Unlike the magnetic particles discussed in the previous section, the magnetic nanoparticles in a ferrofluid do not aggregate in a magnetic field. Due to the uniform suspension of magnetic nanoparticles in the liquid, ferrofluids have been used to induce wetting and fluid deformation. ${ }^{66-70}$ Mixing ferrofluid with an aqueous solution destabilizes the colloidal suspension. Therefore, the unique chemical composition of ferrofluids is not compatible with the environment of an aqueous buffer in many bioanalytical assays. The ferrofluid is used only as the actuator to move the droplet cargo for liquid phase reactions; it does not participate in the solid phase biochemical reactions (Fig. 5). ${ }^{71}$ Another type of fluid used for magnetic digital microfluidics is a solution containing a relatively high concentration of iron ions. ${ }^{72,73}$ This type of liquid, which is sometimes incorrectly referred to as a ferrofluid, is better described as ionic or ferric fluid. Due to the high concentration of iron ions, the droplet responds to an external magnetic field and can be directly manipulated without the addition of actuator magnetic particles to the droplets. However, the high concentration of iron ions is not compatible with most bioanalytical assays, which are sensitive to ionic strength.
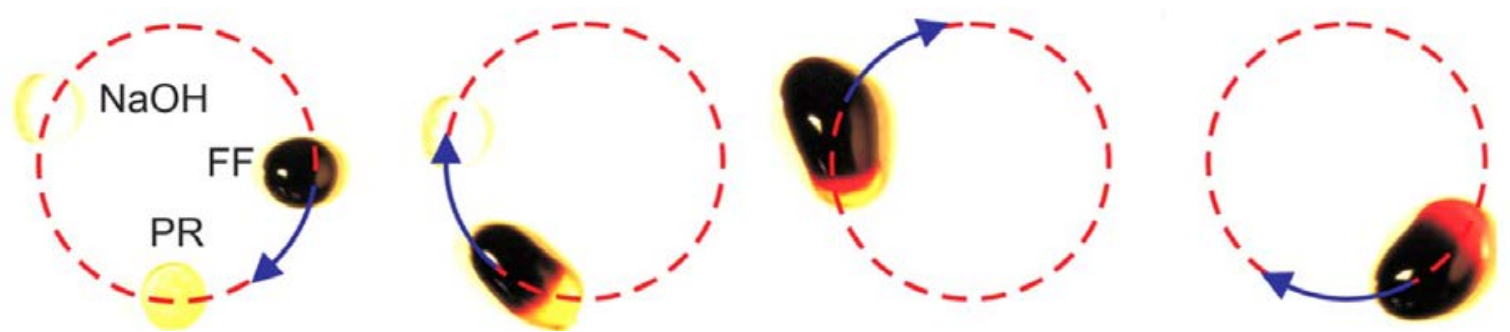

Fig. 5 Magnetic droplet manipulation using a ferrofluid for $\mathrm{pH}$ sensing. FF - ferrofluid, PR - phenol red. Reproduced from Ref. 71 with permission from the American Society of Mechanical Engineering. 


\section{Assisted Magnetic Droplet Manipulation}

Ideally, we could perform all the fluidic operations required for a bioassay by simply manipulating the droplets with a magnet. Transporting and merging the droplets easily allows for liquid transfer and addition. Removing and discarding the waste supernatant is realized by extracting the particles from the droplet. However, particle extraction is not a simple process, depending on many factors such as particle concentration, droplet volume and moving speed. Another important fluidic operation, droplet dispensing, is almost impossible to accomplish using magnetic force alone. As a result, additional features have been introduced to assist in magnetic droplet manipulation. These assistive features generally fall into two categories: physical structures that constrain the droplet and chemical modifications that change the surface properties of the substrate.

The physical structures are primarily designed to facilitate particle extraction. The structures are in the shape of pillars or channels and form a narrow passage (Fig. 6). As the droplet moves close to these structures, it is too large to squeeze through the narrow passage but the magnetic particles can easily split from the droplet and pass through. This type of physical constraint is used in many magnetic digital microfluidic platforms to facilitate particle extraction. Zhang et al. incorporated pillars in a magnetic digital microfluidics-based micro total analysis system that performed integrated DNA extraction and PCR amplification. ${ }^{12}$ Each pair of pillars formed a narrow opening that held the droplet in position while the magnetic particles were extracted from the droplet and moved on. Shin et al. modified this platform for droplet-based sample-to-answer gene mutation detection using high-resolution melting curve analysis. ${ }^{74}$ Chiou et al. showed a similar platform for sample-toanswer molecular diagnostics using electromagnet-based digital microfluidics. ${ }^{9}$ Kim et al. used an array of small pillars to achieve the same goal. ${ }^{53}$ The array of pillars functioned as a sieve that allowed only small magnetic particles to pass. This design was used to perform droplet-based ELISA. Shikida et al. used microchannels to restrict the movement of the droplet. Similar to the narrow opening formed by the pillars, the microchannel stopped the droplet from passing through but allowed magnetic particles to pass, thereby facilitating the particle extraction process. ${ }^{54,75}$ In the examples above, the physical structures were placed on the side of the droplet. In contrast, Shikida et al. created physical structures on top of the droplet. ${ }^{55}$ These overhanging structures created a narrow channel close to the surface. As the droplet moved in, these structures held the droplet in position from the top and the magnetic particles squeezed through the channel at the bottom. Park et al. further developed this design into an array for parallel ELISA-based detection. ${ }^{47}$ 


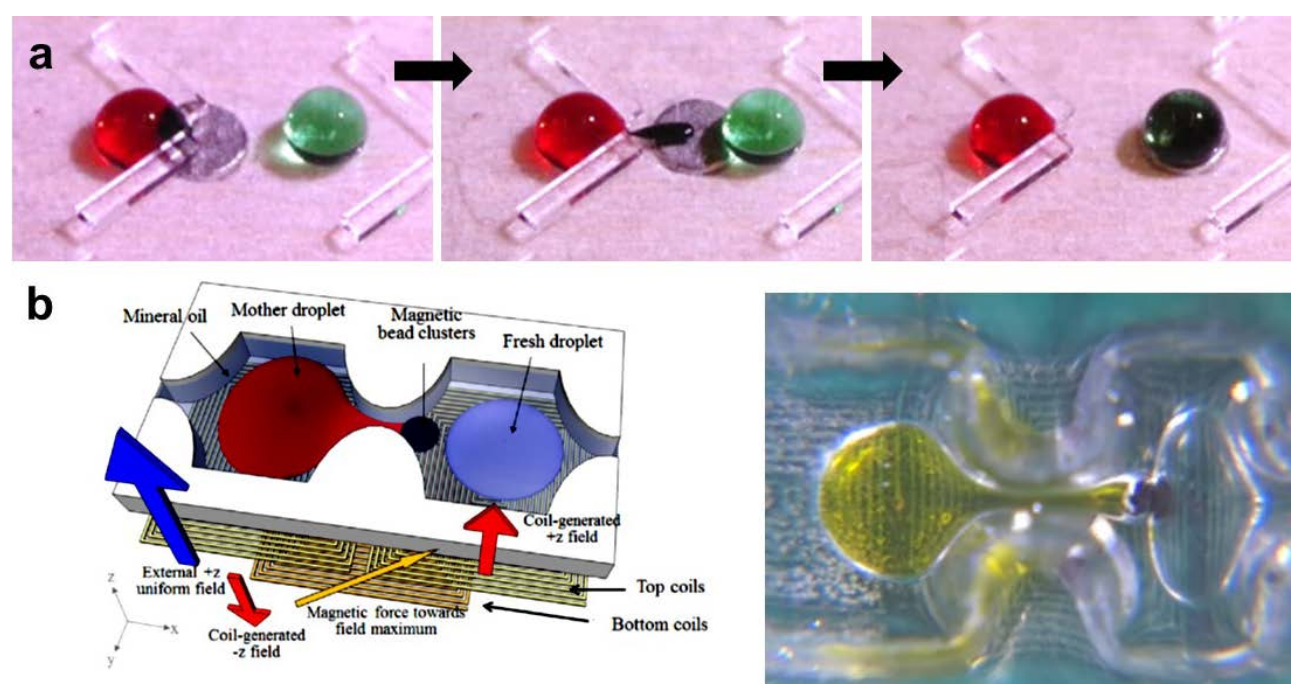

Fig. 6 Physical structure-assisted magnetic digital microfluidics. a) Physical structure-assisted magnetic digital microfluidic platform using a permanent magnet. b) Physical structure-assisted magnetic digital microfluidic platform using an electromagnet. Reproduced from Ref. 12 with permission from the Royal Society of Chemistry and from Ref. 9 with permission from Elsevier.

Similar to physical structures, chemical modifications have also been used to facilitate particle extraction. Zhang et al. modified a Teflon-coated hydrophobic surface with oxygen plasma through a shadow mask to create well-defined hydrophilic traps to immobilize droplets (Fig. 7). ${ }^{13}$ As the droplet moved over these traps, it was anchored to the surface due to high surface tension but the magnetic particles could continue travelling and extract themselves from the droplet. The authors demonstrated droplet-based sample-to-answer molecular diagnostics on this platform using the surface energy traps to facilitate particle extraction. Shi et al. used a similar strategy and created an array of traps to hold many droplets in position for parallel RNA extraction. ${ }^{58}$

Another important application of surface modification is droplet dispensing. As mentioned above, droplet dispensing is virtually impossible using magnetic force alone. Therefore, a secondary force must be introduced to split the droplets to achieve liquid dispensing. In this scenario, the secondary force is the surface tension provided by the surface energy traps. Depending on its size, the trap can either immobilize the entire droplet or withhold only a portion of the droplet. A full range of fluidic operations was demonstrated on a magnetic digital microfluidic platform for the first time, which greatly extended its applicability. ${ }^{13}$ The same paper demonstrated multiplexed sampleto-answer molecular diagnostics by splitting a droplet containing purified DNA into multiple aliquots using surface energy traps. The size of the surface energy trap can be fine-tuned to withhold desired volumes of liquid. Using surface energy traps of various sizes, Zhang et al. successfully dispensed different amounts of an antibiotic solution to create a serial dilution for antimicrobial susceptibility testing. ${ }^{25}$ 


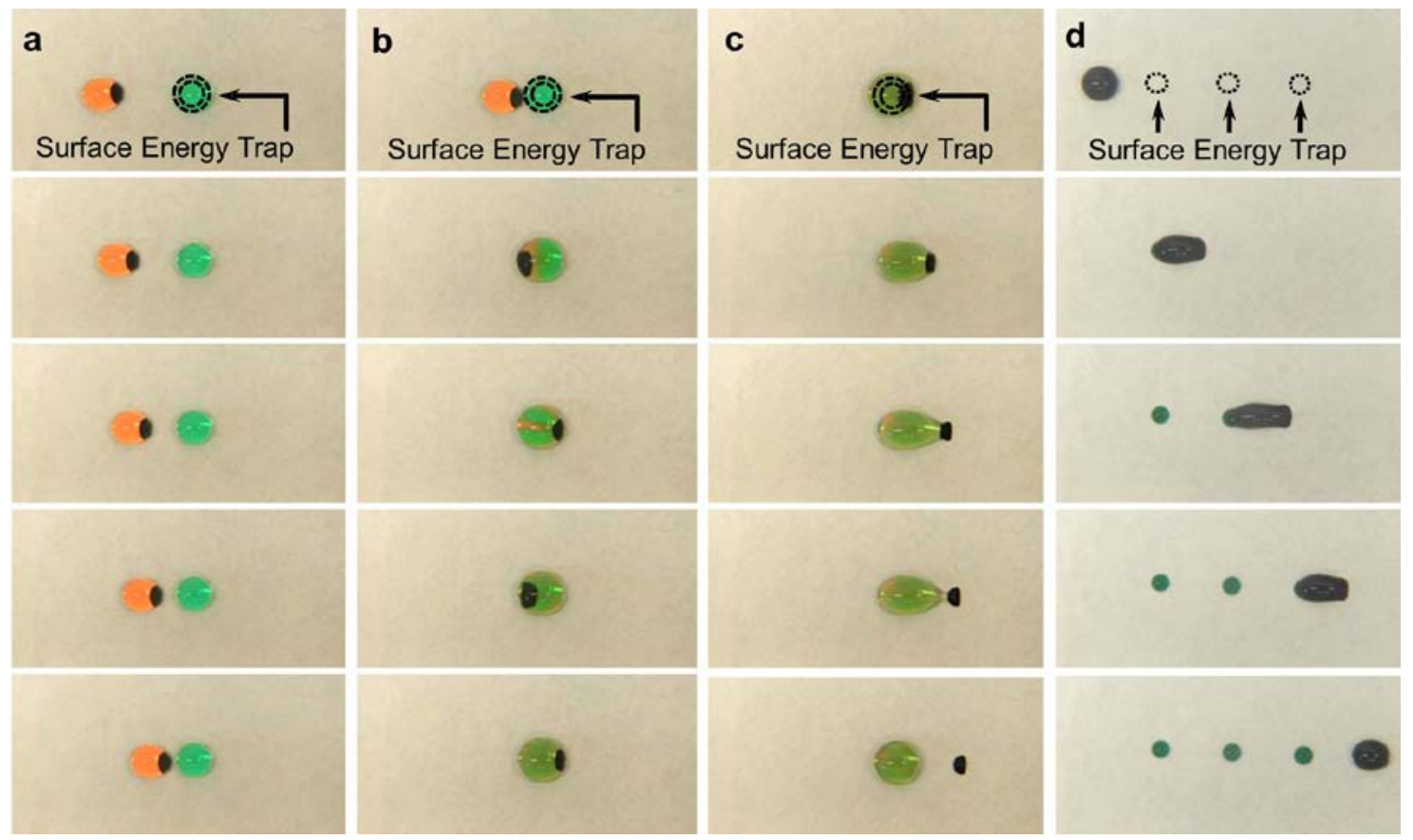

Fig. 7 Surface chemical modification-assisted magnetic digital microfluidics. The surface modification enabled a full range of droplet operations including a) droplet transport, b) droplet fusion and mixing, c) magnetic particle extraction and d) droplet dispensing. Reproduced from Ref. 13 with permission from Wiley.

\section{Magnetic Liquid Marbles}

Liquid marbles are a new type of material that shows great promise for magnetic digital microfluidics. ${ }^{76-79}$ A liquid marble is created by capsulizing a liquid droplet with a layer of hydrophobic or amphiphilic micro/nanoparticles. As the liquid droplet rolls across the particles, the particles adhere to the droplet surface, replacing the liquid-vapour interface with a liquid-solid interface and creating a soft sphere. The particle coating suppresses wetting between the liquid and the surface, and the liquid marble rolls or slides freely on the surface ${ }^{80}$ Using hydrophobic magnetic particles to create liquid marbles allows for magnetic manipulation of the liquid marbles (Fig. 8).

Similar to conventional magnetic particle-based manipulation, a permanent magnet or an electromagnet beneath the surface induces a magnetic force on the liquid marble, which in turn drags the droplet along. Moreover, liquid marbles can float on a liquid surface. ${ }^{48}$ The key difference from conventional droplets is that instead of forming a plug within the droplet, the magnetic particles remain on the surface of the liquid marble. Many studies on liquid marbles have elucidated the underlying physics of liquid marble formation and motion. ${ }^{80}$ Current studies on magnetic liquid marbles focus on novel hydrophobic magnetic particles for liquid marble creation. ${ }^{46,81}$ These 
particles primarily consist of an $\mathrm{Fe}_{3} \mathrm{O}_{4}$ core and a coating with hydrophobic functional groups on the particle surface. As an alternative method for controlling liquid marbles using magnetic force, one can add magnetic particles to the liquid marbles that are capsulated by a nonmagnetic particle coating. ${ }^{48}$ This approach potentially allows for the incorporation of functional magnetic particles for biochemical assays in the liquid marble.

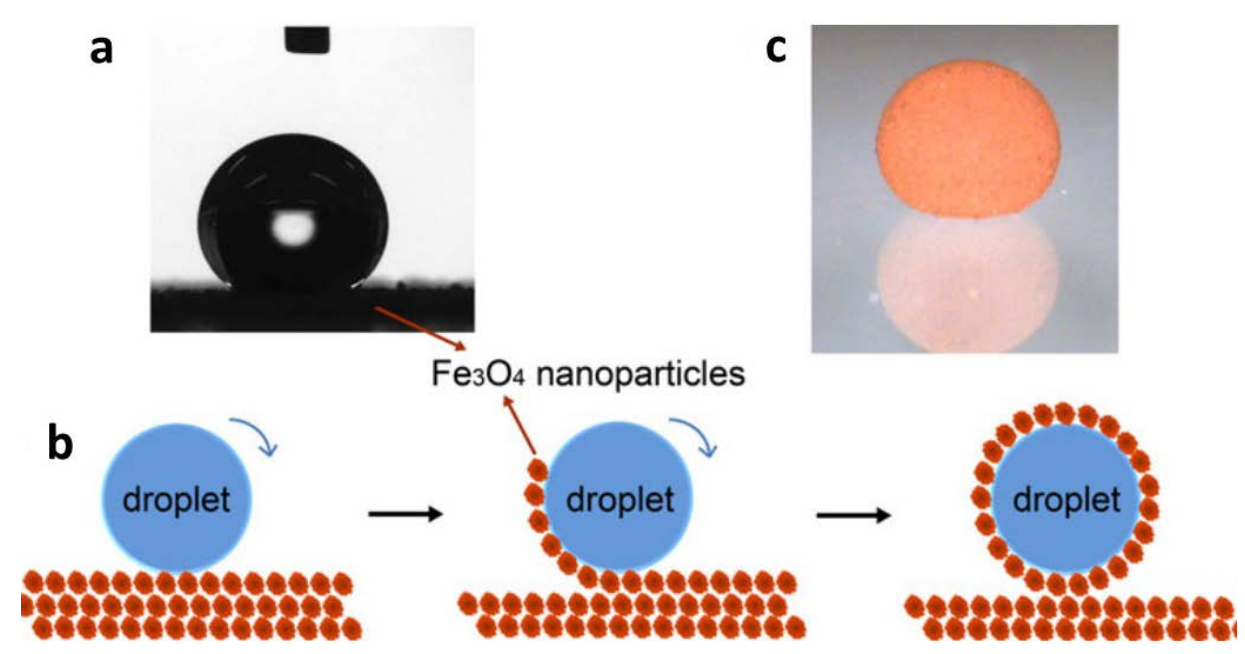

Fig. 8 A magnetic liquid marble. a) Large contact angle of the magnetic liquid marble. b) Principle of a magnetic liquid marble. c) Image of a magnetic liquid marble. Reproduced with permission from Ref. 79 under a Creative Commons Attribution License.

Liquid marbles are of great interest in the field of magnetic digital microfluidics because they are a highly stable droplet and allow for smoother droplet manipulation on a variety of surfaces. Magnetic manipulation remains the primary actuation method. It has also been shown that liquid marbles can reduce evaporation, which has been a major issue in magnetic digital microfluidics. ${ }^{82}$ In conventional magnetic digital microfluidics, silicone oil or mineral oil is often used to cover the droplet to prevent evaporation. ${ }^{10,12,13}$ However, this approach significantly increases the thermal mass and potentially leads to cross contamination.

One major limitation of digital microfluidics for point-of-care applications is the lack of a wellestablished strategy for reagent storage. Unlike in a closed-chamber system where reagents can be easily sealed within the device, reagents for digital microfluidics are often stored off-chip in vials. To solve this problem, we may incorporate a separate pouch or cartridge with pre-metered reagent that can be dispensed on-demand. Liquid marbles provide a potential novel solution. Due to the stability of liquid marbles, reagents may be packed in the form of liquid marble on the substrate with 
a relatively simple physical confinement. The reagents can be released on-demand by stripping off the coating particles using magnetic force. ${ }^{79}$

The high stability of the liquid marble is a double-edged sword. It is much more difficult to merge two liquid marbles, which is an important fluidic operation in many bioassays. Liu et al. demonstrated the coalescence of two liquid marbles by applying an electric potential across the droplets. ${ }^{83}$ Alternatively, one could temporarily "strip" the particle coating to expose the underlying liquid. ${ }^{79}$ This could be achieved by pulling the magnetic particles using a strong magnetic force. As the particles are pulled to the bottom, the top portion of the droplet is stripped, opening a liquid window that could potentially be used for liquid marble merging. Zhang et al. presented a type of "smart" particle with a hydrophobicity that is $\mathrm{pH}$-tuneable. ${ }^{84}$ This type of particle is hydrophobic and forms liquid marbles at neutral $\mathrm{pH}$. At low $\mathrm{pH}$ values, the particle becomes hydrophilic and the liquid marble collapses, exposing the liquid for potential droplet merging. Although the authors claimed that the hydrophobic-hydrophilic transition was fully reversible, liquid marble reformation was not demonstrated.

Another disadvantage of magnetic liquid marbles lies in the fact that the magnetic particles can no longer serve as the solid phase substrate for molecule binding because they remain on the droplet surface. Thus, the magnetic particles play only the role of droplet actuator in the liquid marble, which substantially reduces the applicability of magnetic digital microfluidics. Due to these two main constraints, liquid marbles are currently not well-suited for complex bioassays that require liquid exchange. Only one simple single-tube homogeneous assay for blood typing, which does not require liquid exchange, has been demonstrated using liquid marbles. ${ }^{85}$ Nonetheless, we believe that surface-functionalized magnetic particles can be introduced for molecule binding without interfering with the liquid marble operation because these particles are hydrophilic and will stay within the liquid portion of the marble. ${ }^{48}$

\section{Magnetically Controlled Flexible Substrate for Droplet Manipulation}

Conventionally, magnetic digital microfluidics manipulates droplets on a rigid surface. The rigid surface does not play an additional role other than providing a low-energy substrate to support the droplets. The magnetic force is directly exerted on the droplet through magnetic particles inside the droplet or on the droplet surface. Recently, a new strategy was proposed to manipulate droplets on a flexible substrate. The flexible substrate is easily deformed to create a dent on the surface. The droplets roll towards the dent where the potential energy is the lowest (Fig. 9). By deforming and shifting the location of the dent on the flexible substrate, one can easily control the movement of 
the droplet. One of the most effective methods for deforming a flexible substrate is the use of magnetic force. The flexible substrate, usually composed of elastic polymers, is impregnated with magnetic materials. If a magnet is brought close to the surface, a portion of the substrate is attracted towards the magnet, forming a dent. This strategy was demonstrated in two recent studies. Seo et al. mixed magnetic particles with polydimethylsiloxane (PDMS). ${ }^{86}$ After curing, the resulting flexible substrate was uniformly impregnated with magnetic materials. The authors demonstrated droplet transport and merging on the proposed platform. Biswas et al. reported a different strategy for deforming a flexible substrate using magnetic force. ${ }^{87}$ Instead of uniformly impregnating the elastomer with magnetic particles, they embedded individual steel balls into PDMS. The resulting flexible substrate contained a network of magnetically controllable nodes made of steel balls. When a steel ball was attracted to the permanent magnet below the surface, the substrate deformed and created a dent for droplet control. The authors demonstrated droplet transport and merging by sequentially attracting and releasing the steel balls at different nodes.
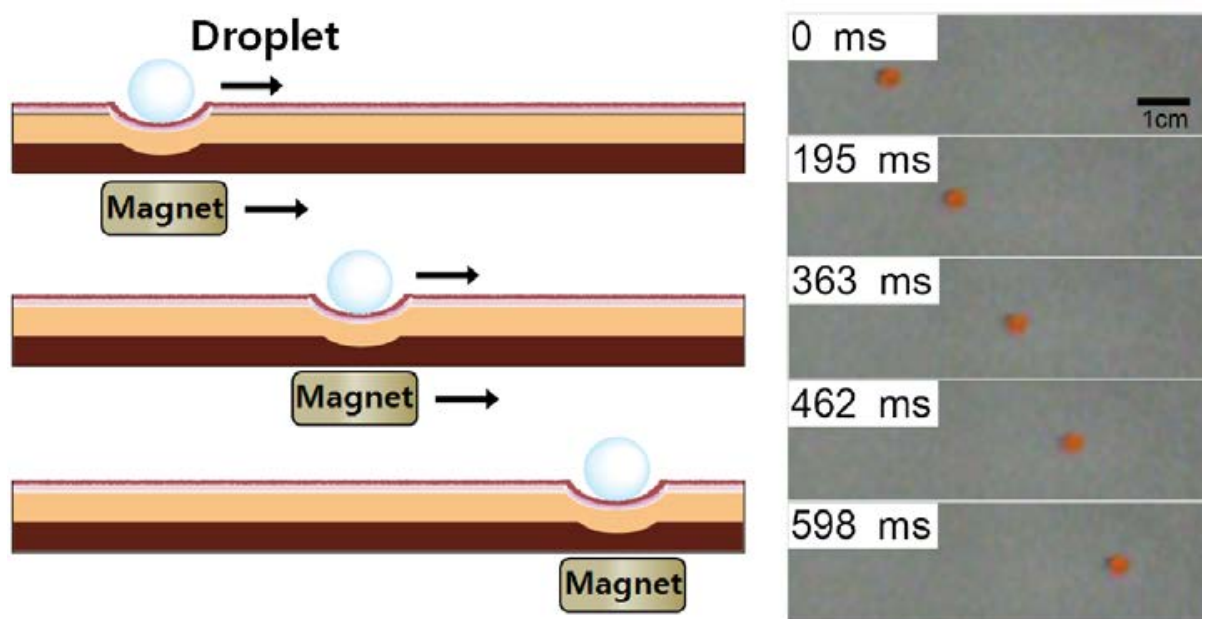

Fig. 9 Droplet manipulation using magnetically deformable flexible substrates. a) Principle of droplet actuation using a magnetically deformable flexible substrate. b) Serial images of a droplet actuated by a magnetically deformable flexible substrate. Adopted from Ref. 86 with permission from Wiley.

Magnetic force has also been used to control the morphology of magnetically susceptible nanostructures on a flexible substrate (Fig. 10). Conventional substrates for magnetic digital microfluidics are smooth and coated with a hydrophobic thin film such as Teflon AF. It is widely known that surface roughness can increase the apparent hydrophobicity and result in a larger contact angle. ${ }^{88}$ The roughness can be engineered by fabricating micro/nanostructures on the surface. If the micro/nanostructures are made of flexible and magnetic materials, the surface roughness can be tuned using magnetic force. This concept was demonstrated by Zhou et al. ${ }^{51}$ In 
their study, magnetic nanoparticles were deposited on a track-etched polycarbonate membrane that served as a sacrificial supporting material. After removing the sacrificial material by plasma etching, the deposition formed rough nanostructures on the surface. The droplet stayed on the surface in the Cassie-Baxter state with a large contact angle. When an external magnetic field was applied, the magnetic nanostructures collapsed, reducing the surface roughness and the apparent contact angle.

Wang et al. also fabricated a magnetically tuneable rough surface. ${ }^{89}$ Nano hair-like structures were grown on an array of micro-sized walls made of elastomers impregnated with magnetic particles. When a permanent magnet was placed on the side, the micro walls tilted towards the magnet. The droplet placed on this substrate stayed on top of the micro walls in the Cassie-Baxter state. When the magnet pulled the wall, the droplet travelled toward the direction of pulling. The droplet motion was not driven by the pressure difference resulting from different contact angles on the two sides of the droplet because the droplet did not move from the side with a larger contact angle to the side with a smaller contact angle as in the case of EWOD. Instead, the droplet moved in the opposite direction. We believed that the side-pulling force resulting from the adhesion of the micro wall played a dominant role in governing the droplet motion.
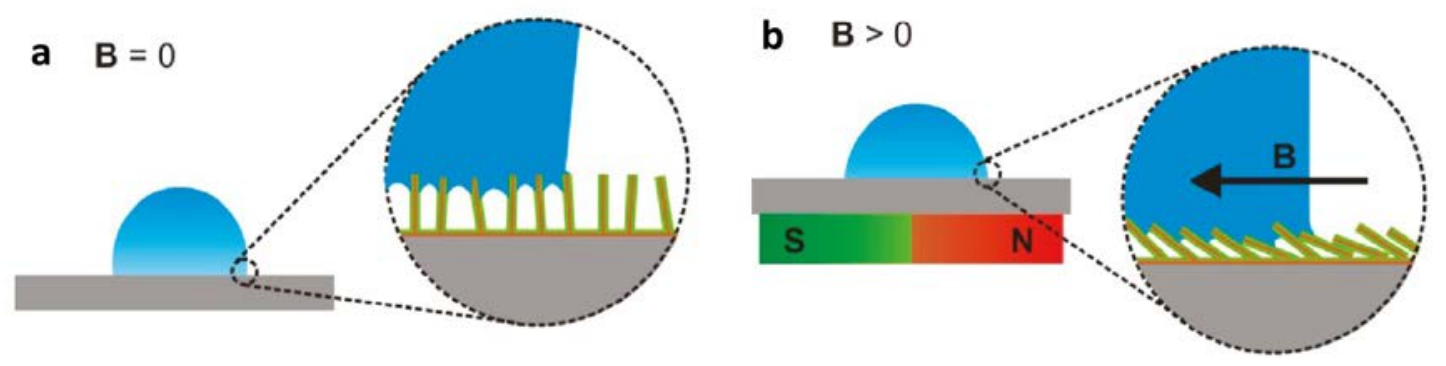

Fig. 10 Magnetically induced droplet wetting for potential liquid dispensing in magnetic digital microfluidics. a) The droplet stays in the Cassie-Baxter state on a patterned rough surface. b) The magnetic force reduces the surface roughness by controlling the magnetically susceptible flexible nanostructures. The droplet changes to the Wenzel state. Adapted from Ref. 51 with permission from the American Chemical Society.

Although manipulating droplets on a magnetically deformable substrate does not require the addition of magnetic particles to the droplet, we can still use magnetic particles as the functional solid substrate for molecule binding. The major disadvantage of this droplet manipulation method lies in the difficulty of creating a magnetically controllable flexible substrate. Most substrates in the examples discussed above entail microfabrication and nanosynthesis techniques, which significantly increase the cost. Even the cost of the simplest steel ball method is large compared to a plain surface with a simple coating. As a main application of magnetic digital microfluidics is sample-to- 
answer analysis for point-of-care diagnostics in low-resource environments, both simple operation and low cost must be considered. 


\section{Conclusions and Future Perspectives}

The present review discusses the unique advantages of magnetic digital microfluidics and examines current challenges of this technology. We investigated various methods of droplet manipulation using magnetic force, ranging from conventional magnetic particle-based actuation to the recent development using ferrofluids and magnetic liquid marbles. We also reviewed several new approaches that use a magnetically controlled flexible substrate for droplet manipulation. As the requirements for droplet-based bioassays are becoming more complex, we also discussed physical structure-assisted and chemical modification-assisted magnetic droplet manipulation. In the last section of the review, we probed several problems associated with magnetic digital microfluidics such as liquid dispensing, evaporation prevention, multiplexing and automation and discussed potential solutions and future research directions.

Although magnetic digital microfluidics is not as well established as EWOD-based digital microfluidics, this emerging field has tremendous potential for bioanalytical and preparative assays due to its simple operation and the unique dual functionality of magnetic particles. Despite recent advances in magnetic droplet manipulation, further work is needed to achieve better droplet control for fluidic operation.

\section{Liquid Dispensing}

One priority is the development of a strategy to dispense droplets of a desired volume that can be moved to a desired location. As mentioned above, the only magnetic digital microfluidic platform capable of droplet dispensing uses a chemically modified surface energy trap. However, the dispensed droplet is anchored to the trap. EWOD can achieve this goal because the wetting property is reversible once the electrical potential is removed. Although surface energy traps also dispense droplets based on the wetting property, the chemical modification is considered permanent and irreversible. The key to this problem is the development of a magnetically activated surface energy trap that is reversible upon removal of the magnetic field. Earlier work by Nguyen et al. showed that the wetting property of a ferrofluid changes in a magnetic field. ${ }^{69}$ Unfortunately, ferrofluids are not compatible with most bioassays. Another interesting work by Zhou et al. elucidated a possible approach (Fig. 10). ${ }^{51}$ The authors used a substrate with magnetically controllable flexible nanostructures. In their original state, the nanostructure supported the droplet with a high degree of surface roughness, resulting in a large contact angle. When a magnetic field was applied, the nanostructures collapsed, reducing the surface roughness and resulting in a smaller contact angle. The process was reversible as the surface roughness was restored once the magnetic field was removed. 


\section{Evaporation Prevention}

Another problem that must be addressed is evaporation of the liquid droplet. This problem is pertinent to all types of digital microfluidics including magnetic force-, EWOD- and SAW-based platforms. As the droplets move on an open surface, they are exposed to the ambient environment and evaporate at a rapid rate. When subjected to heating for certain biochemical reactions such as PCR, the droplet evaporates almost instantly if not covered by oil. However, the use of mineral oil substantially increases the thermal mass and decreases the temperature ramping rate. Furthermore, large quantities of oil are difficult to handle on a miniaturized system and defeat the purpose of microfluidics. Nelson et al. noted a potential solution to this problem. Instead of enclosing the droplets with oil, the author enclosed the droplet in a small pressurized chamber. ${ }^{90}$ The increased ambient pressure combined with high humidity within the enclosure successfully prevented droplet evaporation without adding much thermal mass.

\section{Droplet Manipulation for Multiplexing}

Two improvements required to manage the increasing complexity of bioassays are parallel droplet manipulation and cross-platform droplet transfer. As we push the limit and implement more complex bioassays on magnetic digital microfluidic platforms, multiple reactions must be carried out in parallel for high-throughput analysis. Therefore, multiple droplets must be manipulated concurrently. A few earlier works demonstrated parallel magnetic droplet manipulation. These operations are relatively simple, as all the droplets travel along a single path. ${ }^{53,58}$ To address more complexity and accomplish true multiplexing, individual droplets must be manipulated both separately and collectively.

Complex bioassays also entail a larger number of droplets to perform various reactions. To accommodate this requirement without increasing the device footprint, droplets need to be transferred between different substrates. Zhang et al. demonstrated droplet transfer between different platforms using a chemically modified surface energy trap. ${ }^{13}$ However, once the droplet is transferred to another substrate, it is anchored to the surface energy trap and cannot move to other locations. The magnetically activated wetting discussed in the previous section could potentially solve this problem. Alternatively, other substrate types with a reversible hydrophobic-hydrophilic transition could also serve this purpose. One solution is a flexible substrate with microstructures with surface wetting properties that are alterable by simple stretching. ${ }^{91}$ The stretching alters the surface roughness, thereby changing its hydrophobicity. The authors used this interesting property to transfer droplets across platforms. 
Page 23 


\section{Mixing in the Droplet}

Another desired improvement is the incorporation of features to facilitate fluidic mixing in a droplet. Magnetic digital microfluidics handles microlitres of fluid at the millimetre length scale with Reynolds numbers in the laminar regime. Therefore, mixing in the droplet takes place at a relatively slow rate. The mixing rate can be slightly improved by moving the droplet back and forth with a magnet. However, this approach is not very effective. Two other approaches have been specifically designed to accelerate mixing in a droplet. In one approach, magnetic particles were aligned into chains in a strong uniform magnetic field. ${ }^{92}$ Another alternating magnetic field was applied to induce rotation of the particle chains, which actively mixes the liquid in the droplet. In another approach, a micro magnetic gyromixer is positioned on the droplet surface (Fig. 11). ${ }^{93}$ An alternating magnetic field induces rotation of the gyromixer. The spinning motion allows the gyromixer to balance itself on the droplet surface and actively mix the fluid. Both active mixing approaches are very effective. However, the intricate setup complicates or even interferes with the magnetic droplet operation. Alternatively, surface pattern-enabled passive chaotic mixing is an effective mixing strategy that is widely implemented in conventional microfluidics. Herringbone structures can also be introduced on the surface to induce chaotic mixing in the droplet. This strategy is similar to physical structureassisted magnetic droplet operation.

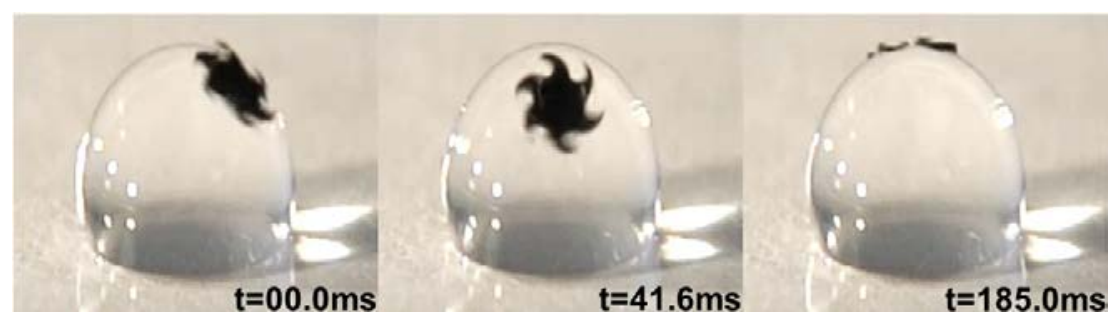

Fig. 11 A magnetic gyromixer in magnetic digital microfluidics. Reproduced from Ref. 93 with permission from Springer.

\section{System Automation}

We need to rethink the automation solution to make magnetic digital microfluidics a pragmatic platform for point-of-care diagnostics in low-resource settings. Currently, two types of automation systems are used for magnetic droplet manipulation. One system uses an electromagnetic micro coil, and the other uses a permanent magnet. The micro coil system is similar to EWOD, which is completely controlled by electrical signals and does not require any moving parts. The permanent magnet system usually requires a motion control system such as a translational stage or rational step motor. The permanent magnet is attached to the moving part and travels together with the stage or motor. Both systems provide automated magnetic droplet manipulation. However, these 
automation solutions are too complicated and expensive for point-of-care applications. They are difficult to set up and require electrical power. Advances in battery technology would make these systems portable and stand-alone. However, point-of-care diagnostics are usually deployed in resource-limited environments, where electricity is considered a luxury item. The simple motion required to control the permanent magnet for droplet manipulation can be easily accomplished using mechanical approaches. Simple springs and wheels in a clock movement provide both precise timing and motion control. A similar strategy can be implemented for magnetic droplet manipulation. Mechanisms can be designed to trigger motion, guide movement, and control the timing of each process. Users need only to wind the spring, and the mechanism can handle the rest in a completely electrical power-free manner.

\section{More Applications of Magnetic Droplets}

The application of magnetic digital microfluidics is still limited. Only two major types of applications have been demonstrated on magnetic digital microfluidic platforms. As mentioned above, the magnetic particles used in magnetic digital microfluidics play two important roles. This unique advantage is well-suited for heterogeneous assays, i.e., assays that require liquid exchange for washing such as ELISA. Another commonly performed heterogeneous assay is solid phase extraction used for DNA or RNA preparation. Magnetic droplet-based sample preparation is often combined with PCR-based detection to achieve sample-to-answer analysis for point-of-care diagnostics. Another application of magnetic digital microfluidics is preparative assays. Radioactive materials, other hazardous chemicals and reagents with a short shelf life can be synthesized on demand. Future work needs to explore other areas that could expand the applications of magnetic digital microfluidics. 


\section{Reference}

1. M. T. Guo, A. Rotem, J. A. Heyman and D. A. Weitz, Lab on a Chip, 2012, 12, 2146-2155.

2. H. N. Joensson and H. Andersson Svahn, Angewandte Chemie International Edition, 2012, 51, 12176-12192.

3. S.-Y. Teh, R. Lin, L.-H. Hung and A. P. Lee, Lab on a Chip, 2008, 8, 198-220.

4. Y. Zhu and Q. Fang, Analytica Chimica Acta, 2013, 787, 24-35.

5. K. Choi, A. H. C. Ng, R. Fobel and A. R. Wheeler, Annual Review of Analytical Chemistry, 2012, 5, 413-440.

6. M. J. Jebrail, M. S. Bartsch and K. D. Patel, Lab on a Chip, 2012, 12, 2452-2463.

7. R. B. Fair, Microfluidics and Nanofluidics, 2007, 3, 245-281.

8. H.-H. Shen, S.-K. Fan, C.-J. Kim and D.-J. Yao, Microfluidics and Nanofluidics, 2014, 16, 965987.

9. C.-H. Chiou, D. J. Shin, Y. Zhang and T.-H. Wang, Biosensors and Bioelectronics, 2013, 50, 9199.

10. J. Pipper, M. Inoue, L. F. P. Ng, P. Neuzil, Y. Zhang and L. Novak, Nature Medicine, 2007, 13, 1259-1263.

11. J. Pipper, Y. Zhang, P. Neuzil and T. M. Hsieh, Angewandte Chemie International Edition, 2008, 47, 3900-3904.

12. Y. Zhang, S. Park, K. Liu, J. Tsuan, S. Yang and T.-H. Wang, Lab on a Chip, 2011, 11, 398-406.

13. Y. Zhang and T. H. Wang, Advanced Materials, 2013, 25, 2903-2908.

14. K. Choi, A. H. C. Ng, R. Fobel, D. A. Chang-Yen, L. E. Yarnell, E. L. Pearson, C. M. Oleksak, A. T. Fischer, R. P. Luoma and J. M. Robinson, Analytical Chemistry, 2013, 85, 9638-9646.

15. C.-Y. Huang, P.-H. Shih, P.-Y. Tsai, I. C. Lee, H.-Y. Hsu, H.-Y. Huang, S.-K. Fan and W. Hsu, Proceedings of the IEEE, 2015, 103, 225-235.

16. A. H. C. Ng, M. D. Chamberlain, H. Situ, V. Lee and A. R. Wheeler, Nature Communications, 2015, 6.

17. M. W. Royal, N. M. Jokerst and R. B. Fair, IEEE Photon. J, 2012, 4, 2126-2135.

18. S. A. Fiel, H. Yang, P. Schaffer, S. Weng, J. A. H. Inkster, M. C. K. Wong and P. C. H. Li, ACS Applied Materials \& Interfaces, 2015, 7, 12923-12929.

19. M. R. Javed, S. Chen, J. Lei, J. Collins, M. Sergeev, H.-K. Kim, C.-J. Kim, R. M. van Dam and P. Y. Keng, Chemical Communications, 2014, 50, 1192-1194.

20. P. Y. Keng, S. Chen, H. Ding, S. Sadeghi, G. J. Shah, A. Dooraghi, M. E. Phelps, N. Satyamurthy, A. F. Chatziioannou and R. M. van Dam, Proceedings of the National Academy of Sciences, 2012, 109, 690-695.

21. E. M. Miller and A. R. Wheeler, Analytical Chemistry, 2008, 80, 1614-1619.

22. W. C. Nelson and C.-J. C. Kim, Journal of Adhesion Science and Technology, 2012, 26, 17471771.

23. S. K. Cho, H. Moon and C.-J. Kim, Journal of Microelectromechanical Systems, 2003, 12, 70-80.

24. D. J. Shin and T.-H. Wang, Annals of Biomedical Engineering, 2014, 42, 2289-2302.

25. Y. Zhang, D. J. Shin and T.-H. Wang, Lab on a Chip, 2013, 13, 4827-4831.

26. U. Lehmann, S. Hadjidj, V. K. Parashar, C. Vandevyver, A. Rida and M. A. M. Gijs, Sensors and Actuators B: Chemical, 2006, 117, 457-463.

27. X. Ding, P. Li, S.-C. S. Lin, Z. S. Stratton, N. Nama, F. Guo, D. Slotcavage, X. Mao, J. Shi and F. Costanzo, Lab on a Chip, 2013, 13, 3626-3649.

28. Z. Guttenberg, H. Müller, H. Habermüller, A. Geisbauer, J. Pipper, J. Felbel, M. Kielpinski, J. Scriba and A. Wixforth, Lab on a Chip, 2005, 5, 308-317.

29. Z. Wang and J. Zhe, Lab on a Chip, 2011, 11, 1280-1285.

30. L. Y. Yeo and J. R. Friend, Annual Review of Fluid Mechanics, 2014, 46, 379-406.

31. P. Y. Chiou, H. Moon, H. Toshiyoshi, C.-J. Kim and M. C. Wu, Sensors and Actuators A: physical, 2003, 104, 222-228.

32. S.-Y. Park, M. A. Teitell and E. P. Y. Chiou, Lab on a Chip, 2010, 10, 1655-1661. 
33. S.-Y. Park and P.-Y. Chiou, Advances in OptoElectronics, 2011, 2011.

34. J. Seo, S.-K. Lee, J. Lee, J. S. Lee, H. Kwon, S.-W. Cho, J.-H. Ahn and T. Lee, Scientific Reports, 2015, 5.

35. M. H. Shamsi, K. Choi, A. H. C. Ng and A. R. Wheeler, Lab on a Chip, 2014, 14, 547-554.

36. A. H. C. Ng, K. Choi, R. P. Luoma, J. M. Robinson and A. R. Wheeler, Analytical Chemistry, 2012, 84, 8805-8812.

37. C.-Y. Huang, P.-Y. Tsai, I. C. Lee, H.-Y. Hsu, H.-Y. Huang, S.-K. Fan, D.-J. Yao, C.-H. Liu and W. Hsu, Biomicrofluidics, 2016, 10, 011901.

38. S. K. Cho and Y. Zhao, Lab on a Chip, 2007, 7, 490-498.

39. G. J. Shah, A. T. Ohta, E. P. Y. Chiou and M. C. Wu, Lab on a Chip, 2009, 9, 1732-1739.

40. R. Mariella Jr, Biomedical Microdevices, 2008, 10, 777-784.

41. S. Park, Y. Zhang, S. Lin, T.-H. Wang and S. Yang, Biotechnology Advances, 2011, 29, 830-839.

42. P. Rådström, R. Knutsson, P. Wolffs, M. Lövenklev and C. Löfström, Molecular Biotechnology, 2004, 26, 133-146.

43. T. Taniguchi, T. Torii and T. Higuchi, Lab on a Chip, 2002, 2, 19-23.

44. M. Abdelgawad, S. L. S. Freire, H. Yang and A. R. Wheeler, Lab on a Chip, 2008, 8, 672-677.

45. Z. Long, A. M. Shetty, M. J. Solomon and R. G. Larson, Lab on a Chip, 2009, 9, 1567-1575.

46. X. Lin, W. Ma, H. Wu, S. Cao, L. Huang, L. Chen and A. Takahara, Chemical Communications, 2016, 52, 1895-1898.

47. M. C. Park, M. Kim, G. T. Lim, S. M. Kang, S. S. A. An, T. S. Kim and J. Y. Kang, Lab on a Chip, 2016, 16, 2245-2253.

48. M. K. Khaw, C. H. Ooi, F. Mohd-Yasin, R. Vadivelu, J. St John and N.-T. Nguyen, Lab on a Chip, 2016, 16, 2211-2218.

49. U. Lehmann, C. Vandevyver, V. K. Parashar and M. A. M. Gijs, Angewandte Chemie International Edition, 2006, 45, 3062-3067.

50. E. Bormashenko, R. Pogreb, Y. Bormashenko, A. Musin and T. Stein, Langmuir, 2008, 24, 12119-12122.

51. Q. Zhou, W. D. Ristenpart and P. Stroeve, Langmuir, 2011, 27, 11747-11751.

52. L. Mats, R. Young, G. T. T. Gibson and R. D. Oleschuk, Sensors and Actuators B: Chemical, 2015, 220, 5-12.

53. J. A. Kim, M. Kim, S. M. Kang, K. T. Lim, T. S. Kim and J. Y. Kang, Biosensors and Bioelectronics, 2015, 67, 724-732.

54. M. Shikida, K. Takayanagi, H. Honda, H. Ito and K. Sato, Journal of Micromechanics and Microengineering, 2006, 16, 1875.

55. M. Shikida, K. Takayanagi, K. Inouchi, H. Honda and K. Sato, Sensors and Actuators B: Chemical, 2006, 113, 563-569.

56. T. Ohashi, H. Kuyama, N. Hanafusa and Y. Togawa, Biomedical Microdevices, 2007, 9, 695702.

57. M. Okochi, H. Tsuchiya, F. Kumazawa, M. Shikida and H. Honda, Journal of Bioscience and Bioengineering, 2010, 109, 193-197.

58. X. Shi, C.-H. Chen, W. Gao, S.-h. Chao and D. R. Meldrum, Lab on a Chip, 2015, 15, 1059-1065.

59. H. Tsuchiya, M. Okochi, N. Nagao, M. Shikida and H. Honda, Sensors and Actuators B: Chemical, 2008, 130, 583-588.

60. A. Beyzavi and N.-T. Nguyen, Journal of Micromechanics and Microengineering, 2008, 18, 095018.

61. N.-T. Nguyen, K. M. Ng and X. Huang, Applied Physics Letters, 2006, 89, 052509.

62. A. Rida, V. Fernandez and M. A. M. Gijs, Applied Physics Letters, 2003, 83, 2396-2398.

63. A. Beyzavi and N.-T. Nguyen, Journal of Physics D: Applied Physics, 2008, 42, 015004.

64. A. Beyzavi and N.-T. Nguyen, Journal of Micromechanics and Microengineering, 2009, 20, 015018.

65. USA Pat., US3215572 A, 1965. 
66. S. Manukyan and M. Schneider, Langmuir, 2016, 32, 5135-5140.

67. N.-T. Nguyen, Langmuir, 2013, 29, 13982-13989.

68. N.-T. Nguyen, A. Beyzavi, K. M. Ng and X. Huang, Microfluidics and Nanofluidics, 2007, 3, 571-579.

69. N.-T. Nguyen, G. Zhu, Y.-C. Chua, V.-N. Phan and S.-H. Tan, Langmuir, 2010, 26, 12553-12559.

70. N.-T. Nguyen, Microfluidics and Nanofluidics, 2012, 12, 1-16.

71. W. H. Koh, K. S. Lok and N.-T. Nguyen, Journal of Fluids Engineering, 2013, 135, 021302.

72. V. Misuk, A. Mai, K. Giannopoulos, F. Alobaid, B. Epple and H. Loewe, Lab on a Chip, 2013, 13, 4542-4548.

73. Z.-G. Guo, F. Zhou, J.-C. Hao, Y.-M. Liang, W.-M. Liu and W. T. S. Huck, Applied physics Letters, 2006, 89, 081911.

74. D. J. Shin, Y. Zhang and T.-H. Wang, Microfluidics and Nanofluidics, 2014, 17, 425-430.

75. M. Shikida, N. Nagao, R. Imai, H. Honda, M. Okochi, H. Ito and K. Sato, Journal of Micromechanics and Microengineering, 2008, 18, 035034.

76. P. Aussillous and D. Quéré, Nature, 2001, 411, 924-927.

77. G. McHale and M. I. Newton, Soft Matter, 2011, 7, 5473-5481.

78. Y. Zhao, Z. Xu, H. Niu, X. Wang and T. Lin, Advanced Functional Materials, 2015, 25, 437-444.

79. Y. Zhao, Z. Xu, M. Parhizkar, J. Fang, X. Wang and T. Lin, Microfluidics and Nanofluidics, 2012, 13, 555-564.

80. C. H. Ooi and N.-T. Nguyen, Microfluidics and Nanofluidics, 2015, 19, 483-495.

81. Y. Zhao, J. Fang, H. Wang, X. Wang and T. Lin, Advanced Materials, 2010, 22, 707-710.

82. M. Dandan and H. Y. Erbil, Langmuir, 2009, 25, 8362-8367.

83. Z. Liu, X. Fu, B. P. Binks and H. C. Shum, Soft Matter, 2016.

84. L. Zhang, D. Cha and P. Wang, Advanced Materials, 2012, 24, 4756-4760.

85. T. Arbatan, L. Li, J. Tian and W. Shen, Advanced Healthcare Materials, 2012, 1, 80-83.

86. K. S. Seo, R. Wi, S. G. Im and D. H. Kim, Polymers for Advanced Technologies, 2013, 24, 10751080.

87. S. Biswas, Y. Pomeau and M. K. Chaudhury, Langmuir, 2016, 32, 6860-6870.

88. R. N. Wenzel, The Journal of Physical Chemistry, 1949, 53, 1466-1467.

89. L. Wang, M. Zhang, W. Shi, Y. Hou, C. Liu, S. Feng, Z. Guo and Y. Zheng, Scientific Reports, 2015, 5.

90. W. C. Nelson, M. Yen, P. Y. Keng, R. M. Van Dam and C. J. Kim, presented in part at the 16th International Solid-State Sensors, Actuators and Microsystems Conference, Beijing, China, 2011.

91. L. Wang, Z. Wang, T. Wu, Y. Zhao and Y. Suzuki, presented in part at the $19^{\text {th }}$ International Conference on Miniaturized Systems for Chemistry and Life Sciences, Gyeongju, Korea, 2015.

92. T. Roy, A. Sinha, S. Chakraborty, R. Ganguly and I. K. Puri, Physics of Fluids (1994-present), 2009, 21, 027101.

93. Y. Zhang and T.-H. Wang, Microfluidics and Nanofluidics, 2012, 12, 787-794. 